\title{
Research Article \\ Off-Center Error Correction of AMR Yokeless Current Transducer
}

\begin{abstract}
Pavel Mlejnek and Pavel Ripka
Department of Measurement, Faculty of Electrical Engineering, Czech Technical University in Prague, Technicka 2, 16627 Prague 6, Czech Republic
\end{abstract}

Correspondence should be addressed to Pavel Ripka; ripka@fel.cvut.cz

Received 22 June 2017; Revised 15 August 2017; Accepted 7 September 2017; Published 17 October 2017

Academic Editor: Lucio Pancheri

Copyright (C) 2017 Pavel Mlejnek and Pavel Ripka. This is an open access article distributed under the Creative Commons Attribution License, which permits unrestricted use, distribution, and reproduction in any medium, provided the original work is properly cited.

\begin{abstract}
We present a method of calibration and error correction of the AMR yokeless current transducer consisting of a circular array of eight anisotropic magnetoresistors (AMR) with one feedback compensation loop. The main sources of errors are the nonidentical parameters of AMR sensors and off-center position of the measured current. It is well known that AMR sensors from the same batch have $2 \%$ spread of the sensitivity; we found that the variation of the factor of the internal compensation coil is the same. We developed a novel calibration process using the readings of individual residual uncompensated voltages of the AMRs. The position of the current inside the measurement hole is estimated from the individual voltages considering the influence of external DC magnetic field such as the Earth's field. During the calibration phase, the sensor outputs are measured for several positions of the current conductor inside the measuring hole. As a result of calibration the lookup table of error corrections is calculated and stored in the memory, and then these values are used for the correction during the measurement of the unknown current. This procedure reduces the off-center error from $0.4 \%$ to $0.06 \%$.
\end{abstract}

\section{Introduction}

Contactless DC/AC electric current transducers usually have magnetic circuit or yoke. The high permeability of the yoke material concentrates magnetic field into the yoke and makes the transducer reading less dependent on the position of the measured conductor. The yoke also works as a shielding against external magnetic fields including those caused by external currents [1]. Detection of the yoke DC flux is made either by DC magnetic sensor inserted into the air gap in the yoke or by using fluxgate effect [2]. Despite the advantages of the magnetic yoke, industry often demands yokeless current transducers to reduce size, weight, and cost. The second motivation for a yokeless solution may be to increase the safety distance from the high-voltage conductor. And the third reason for avoiding the use of ferromagnetic materials is the danger of saturation by overcurrent [3]. If the DC operation is not required, Rogowski coils or similar devices can be used [4]. Optical fiber current sensors also work without yoke; however, these devices have poor DC stability [5].
The current range of the integrated yokeless current transducers is technologically limited to units of Amps [6]. Commercially available yokeless high current transducers use two discrete sensors on both sides of the bus bar [7]. The main disadvantage of using a single pair of sensors is that the suppression of the external currents by the gradiometric sensor is low. Using four sensors around the conductor reduces this error to $6 \%$ in the close vicinity [8]. A circular sensor array with 6 and more sensors around the conductor approximates better the closed line integral in Ampere's law. The circular sensor array improves the resistance against the position of the measured conductor [9] and also against the external magnetic field and current in external conductors [10]. Both errors decrease with increasing number of sensors, and eight sensors are considered as an optimum number.

Almost every magnetic field sensor can be used in circular current transducers. GMR sensors suffer from poor linearity and also have limited temperature stability [11, 12]. Low sensitivity and poor temperature stability of the sensitivity 


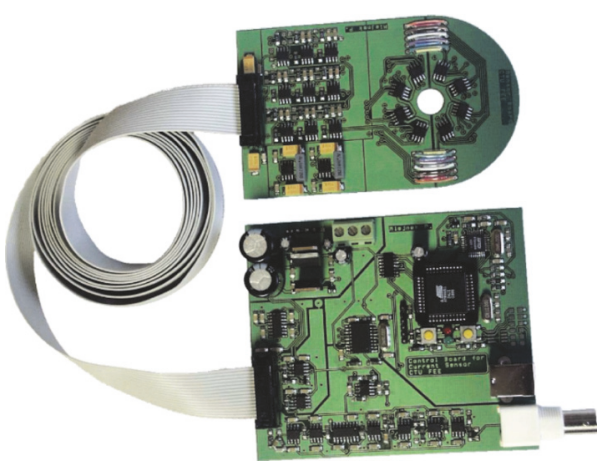

(a)

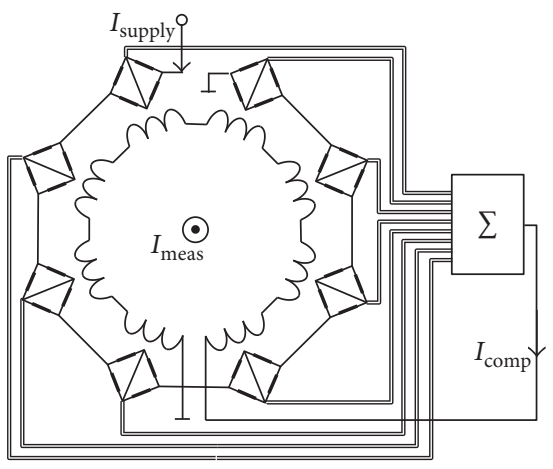

(b)

FIGURE 1: Circular AMR current transducer [16] and wiring of compensation coils and measurement bridges of AMR.

and offset are also disadvantage of magnetoelectric sensors [13]. Hall sensors have very large field range, but they have very poor DC offset temperature stability and limited linearity [3].

Recently introduced integrated fluxgate sensors (DRV425 by Texas Instruments) have excellent offset stability and linearity, $2 \mathrm{mT}$ range, but higher noise than AMR sensors, which have a lower range of typically $200 \mu \mathrm{T}[14,15]$. In conclusion, Hall sensors are the best solution for kA currents; integrated fluxgate sensors are suitable for the current range of $100 \mathrm{~A}$ and AMR sensors can be used for $10 \mathrm{~A}$ range with $\mu \mathrm{A}$ resolution.

In our previous paper [16] we introduced the methods how to improve the accuracy of the yokeless current transducer (Figure 1) with a circular array of anisotropic magnetoresistors (AMR). Eight KMZ51 [17] magnetic field sensors are arranged around the measurement hole. The measured conductor is positioned inside the hole perpendicularly to the sensor plane. The number of sensors was chosen as a compromise between the complexity of the device and the suppression of error caused by the off-center position of the current [9]. All the sensors are placed on a circle whose radius is $R=12.7 \mathrm{~mm}$ and the measurement hole is $10 \mathrm{~mm}$ in diameter.

All eight KMZ51 sensors are synchronously flipped. Flipping is periodical remagnetization of the sensor. The output of the flipped sensor should be processed by the demodulator. Flipping improves dramatically the sensor offset stability, resistance to perpendicular fields (cross field effect), and resistance to field shocks.

All AMR bridges are connected in series and supplied with a constant current from a single current source (see Figure 1). The differences between the bridge resistances are one source of the differences between the sensor sensitivities. Each bridge output is amplified by an instrumentation amplifier, and these outputs are summed and synchronously demodulated. This signal is converted to the compensation current that is used for all sensors. Compensation current flows through planar compensation coils integrated on AMR sensor. All these coils are connected in series (see Figure 1). Serial connection means that the sensors are not individually feedback compensated, they are only partly compensated by the compensation current derived from the average output and their output is not true zero.

In the real case, the spread of the sensor parameters is significant even within the sensors from the same batch. Main parameters that can affect the error of current estimation are sensitivity $S$ and its field dependence, bridge resistance $R_{\text {bridge, }}$, and the compensation coil field factor $A_{\text {comp }}$. Differences in compensation coil resistance $R_{\text {comp }}$ have in our case no effect because the compensation coils of AMR are connected in series.

The sensitivity $S$ influences the accuracy of current transducer only in the case the measured magnetic field is not well compensated by the compensation coil. The poor compensation can be caused by noncentered measured current and/or by nonidentical compensation coil field factor. The closed feedback loop ensures that the sum of bridge voltages is zero but not that each AMR sensor works as a zero indicator.

In this paper, we show that errors mentioned above can be suppressed by the reading of individual outputs of AMR sensors and digital processing of that data.

\section{Theoretical Background}

As it was presented in our previous paper [16] the measurement error of AMR current transducer is lower than $0.01 \%$ in the range $\pm 11 \mathrm{~A}$ when the current carrying conductor is placed exactly in the center of the transducer. However, this cannot be guaranteed in the real application such as measuring the current in cables. Therefore, it is necessary to know the error of current estimation when the conductor moves inside the measurement hole.

Measured current $I$ flowing through a straight conductor creates a circular magnetic field $H$ that can be calculated at a distance $a$ as

$$
H=\frac{I}{2 \pi \cdot a} .
$$

Let us assume the circular array of AMR sensors like in the Figure 2 . There are eight symmetrically concentrically placed AMRs. 


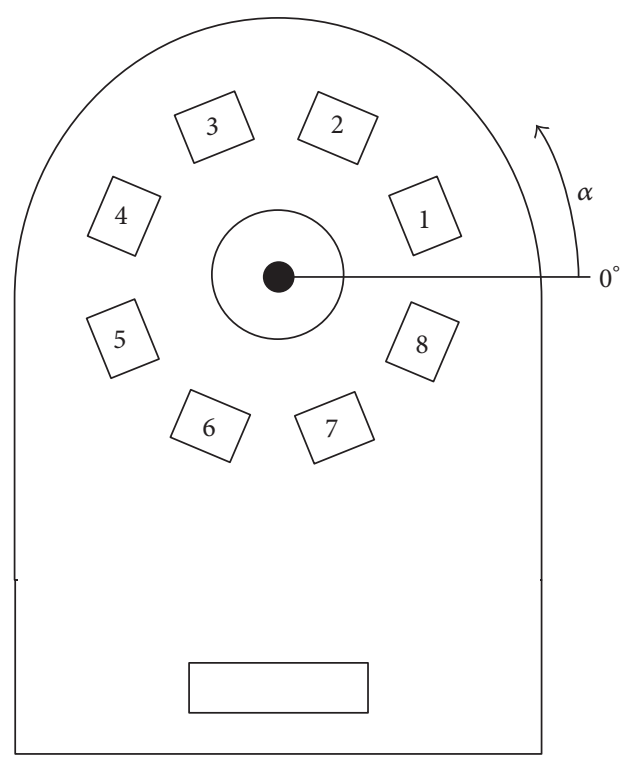

FIGURE 2: Definition of direction and sensor numbering.

The magnetic field from current with arbitrary position (inner or outer) measured by each AMR sensor can be derived according to the Figure 3 where $H_{i}$ is the magnetic field generated by the current $I$ at a distance $a_{i}$ and $H_{M i}$ is the component of the magnetic field along the sensitivity axis of the $i$ th sensor. The current is located at the distance $r$ from the center of the device and angle $\alpha$ from the reference direction. The AMR sensors are placed at the distance $R$ from the center of the device and angle $\beta_{i}$ from the reference direction.

Magnetic field measured by $i$ th AMR sensor is

$$
H_{M i}(r, \alpha)=H_{i} \cdot \cos \gamma_{i}=\frac{I}{2 \pi \cdot a_{i}} \cdot \cos \gamma_{i} .
$$

Distance $a_{i}$ and angle $\gamma_{i}$ can be calculated from cosine formula, and then the magnetic field is defined:

$$
H_{M i}(r, \alpha)=\frac{I}{2 \pi} \cdot \frac{R-r \cdot \cos \left(\beta_{i}-\alpha\right)}{R^{2}+r^{2}-2 \cdot R \cdot r \cdot \cos \left(\beta_{i}-\alpha\right)} .
$$

The compensation current holds the zero of the sum of magnetic fields (if we assume identical sensitivity and compensation coil factor $A_{\text {comp }}$ for each sensor):

$$
\sum_{i=0}^{n}\left(H_{M i}(r, \alpha)-I_{\text {comp }} \cdot A_{\text {comp }}\right)=0,
$$

where $n$ is the number of sensors (eight in our case).

The output voltage of individual AMR sensor can be expressed as

$$
\begin{array}{r}
U_{M i}=\text { Sense } \cdot\left(\frac{I}{2 \pi} \cdot \frac{R-r \cos (\beta-\alpha)}{R^{2}+r^{2}-2 R r \cos (\beta-\alpha)}\right. \\
\left.-A_{\text {comp }} \cdot I_{\text {comp }}+H_{\text {Earth }} \sin (\delta-\beta)\right)+ \text { Off set, }
\end{array}
$$

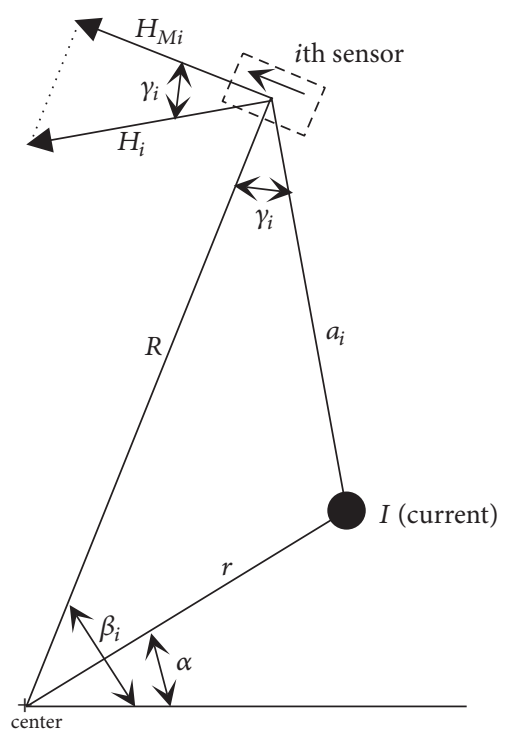

FIGURE 3: Arrangement of the sensor and measured current.

where $H_{\text {Earth }}$ is the magnitude of the vector of magnetic field of the Earth and $\delta$ is the angle between this vector and the reference direction.

The measurement error is defined as

$$
\delta_{n}(r, \alpha)=\frac{\sum_{i=0}^{n} H_{M i}(r, \alpha)-\sum_{i=0}^{n} H_{M i}(0)}{\sum_{i=0}^{n} H_{M i}(0)},
$$

where $H_{M i}(0)$ is the magnetic field from the measured current placed in the center of the device.

This formula is equivalent to

$$
\delta(r, \alpha)=\frac{I(r, \alpha)-I(0)}{I(0)}
$$

where $I(r, \alpha)$ is the estimated current located at distance $r$ from the center and with the angle $\alpha$ and $I(0)$ is the estimated current placed at the center.

The maximal error (positive or negative) strongly depends on a number of sensors and the distance from the center. The off-center error for current, in the distance of $5 \mathrm{~mm}$ from the center, is less than $0.06 \%$ [9] for eight sensors, assuming the same sensitivity and compensation coil factor for all AMR sensors.

\section{Variation of AMR Sensor Parameters}

There are three main parameters of AMR that affect the accuracy of the current measurement. These parameters are the supply bridge voltage, the sensitivity of the AMR, and factor of compensation coils (CC). In the following analysis, we suppose that the sensor offset and the effect of the Earth's field are compensated by measurement without current. In this section, we measure and calculate these parameters to identify spread within sensors from the same batch.

The bridge voltage depends on the bridge resistance. In our case, the voltages of particular sensors are placed in Table 1 . The voltages were measured directly on the device. 
TABLE 1: Real parameters of AMR.

\begin{tabular}{lccccccccc}
\hline & & Sensor 1 & Sensor 2 & Sensor 3 & Sensor 4 & Sensor 5 & Sensor 6 & Sensor 7 & Sensor 8 \\
\hline \multirow{2}{*}{ Bridge voltage } & $(\mathrm{V})$ & 5,117 & 5,357 & 5,554 & 5,357 & 5,103 & 5,263 & 5,542 & 5,177 \\
& $\operatorname{dev}(\%)$ & $-3,6 \%$ & $0,9 \%$ & $4,6 \%$ & $0,9 \%$ & $-3,9 \%$ & $-0,9 \%$ & $4,4 \%$ & $-2,5 \%$ \\
\hline \multirow{2}{*}{ Sensor offset } & $(\mu \mathrm{V})$ & $-1,96$ & 16,89 & $-27,16$ & 13,97 & $-1,05$ & 14,38 & 4,59 & 17,83 \\
& $(\mathrm{~A} / \mathrm{m})$ & $-0,019$ & 0,156 & $-0,248$ & 0,128 & $-0,010$ & 0,138 & 0,042 & 0,170 \\
\hline \multirow{2}{*}{ Sensitivity } & $(\mathrm{mV} / \mathrm{V}) /(\mathrm{kA} / \mathrm{m})$ & 20,21 & 20,25 & 19,72 & 20,32 & 20,26 & 19,79 & 19,69 & 20,26 \\
& $\operatorname{dev}(\%)$ & 0,72 & 0,93 & $-1,70$ & 1,30 & 1,00 & $-1,38$ & $-1,84$ & 0,97 \\
\hline \multirow{2}{*}{ Factor comp. coils } & $(\mathrm{A} / \mathrm{m}) / \mathrm{mA}$ & 23,43 & 23,29 & 23,37 & 23,07 & 23,47 & 23,60 & 23,42 & 23,32 \\
& $\operatorname{dev}(\%)$ & 0,26 & $-0,33$ & 0,00 & $-1,29$ & 0,42 & 0,98 & 0,21 & $-0,24$ \\
\hline \multirow{2}{*}{ Angular alignment } & $(\mathrm{deg})$ & 0,002 & 0,002 & 0,006 & 0,000 & 0,002 & 0,002 & 0,006 & 0,000 \\
\hline
\end{tabular}

Next, the individual voltages were measured inside magnetic shielding to validate the sensors offset.

The feedback loop was disconnected, and the transducer was placed into the Helmholtz coils to estimate sensitivities and factors of compensation coils. The Helmholtz coils were positioned perpendicular to the magnetic field of the Earth. Coils created magnetic field $150 \mathrm{~A} / \mathrm{m}$. Individual AMR sensors were placed in this field, and the output voltage was measured. After that, the compensation current was adjusted to compensate the magnetic field generated by Helmholtz coil. The sensitivity and compensation coil factor (related to the bridge voltage as in [16]) were computed from the measured values (Table 1), where we also indicate their deviations from the average value.

From Table 1 we can draw the following conclusions:

(i) The sensor offsets are very small thanks to flipping. As a result, the horizontal component of the Earth's field $(16 \mathrm{~A} / \mathrm{m}$ in the Central Europe) and from man-made sources such as permanent magnets and DC currents is the dominant source of the zero readings.

(ii) Errors caused by angular misalignment of individual sensors are negligible.

(iii) Variation of the bridge resistance of individual sensors from the same batch is $\pm 4 \%$. We believe that the main source of this variation is variable thickness of the sputtered magnetic layer.

(iv) The open-loop sensitivity of individual sensors corrected for the difference in applied voltage (i.e., expressed in $\mathrm{mV} / \mathrm{V}$ units) depends on the size of the magnetoresistive effect and on demagnetization, which is a function of thickness and permeability. These factors are difficult to control even within the same wafer. We observed a variation of $\pm 2 \%$.

(v) We obtain similar results with the measurement of the factor of compensation coils. In this case, the variation is in the range of $\pm 1.5 \%$. To explain such large variation, we should consider that the compensation coil is flat and the generated field depends not only on the coil geometry but also on the properties of the ferromagnetic layer. Surprisingly we could not find similar measurement in the available literature.

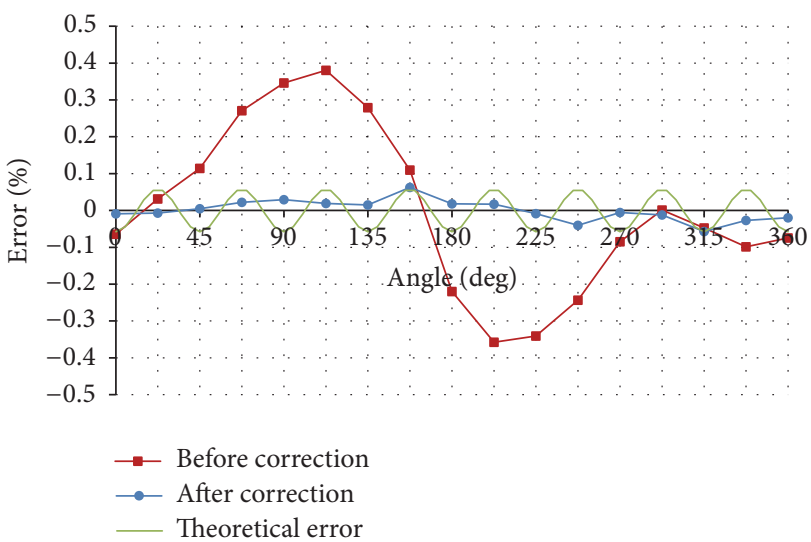

Figure 4: Measurement error due to off-center current at the edge of $10 \mathrm{~mm}$ diameter hole (red square: before correction, blue circle: after correction, green: theoretically computed error).

\section{Measurement of Off-Center Current}

In Section 2, the theoretical off-centered measurement error was derived. According to the result, the real measurement was done. The results are shown in Figure 4, red (square) plot. The error is almost one order of magnitude larger than the theoretically calculated error of $0.06 \%$ for eight ideal sensors (Figure 4, green plot). The problem is caused by the fact that, due to the shared compensation current for all sensors, the measured magnetic field (generated by the current) is not fully compensated for each particular sensor. Therefore, all sensors work at the nonzero magnetic field and therefore the different sensitivities, compensation coil factors, and additional imperfections affect the performance of the device.

Correction of this error can be made using sensor parameters measured in Section 3. However, such correction is not practical for industrial applications as it requires magnetic shielding and rather complicated calibration process. Therefore, we developed an alternative calibration procedure which can be repeated periodically before the use of the current transducer. Our new calibration method is based on 


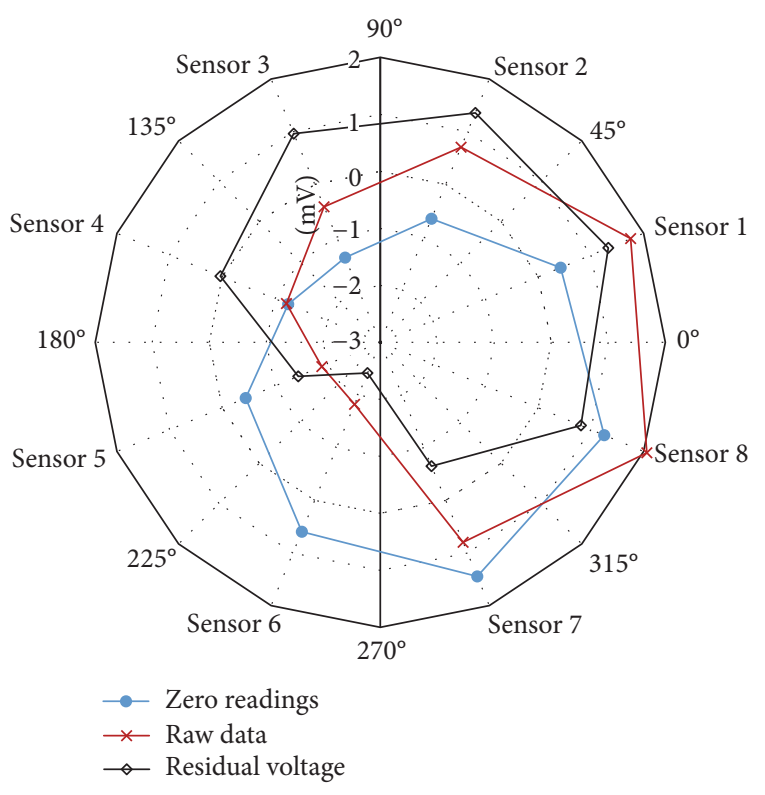

FIGURE 5: The residual output voltage of the AMR sensors with the off-center current at the angle $235^{\circ}$.

an intentional movement of the transducer with respect to the measured current.

\section{New Correction Method}

Our new method is based on processing the residual outputs of the individual AMR sensor. First, we make zero correction without the measured current. As a second step we take the reading measure with measured current, and from the residual sensor voltages, we estimate the position of the current. Finally, we perform the correction.

5.1. Reading of the Individual AMR Residual Outputs. The AMR current sensor zero output (sensor output for the zero measured current) is affected by the offset of the individual AMR sensors and by the external fields (which come from the Earth magnetic field and human-made field sources such as near electric currents) as was presented in [16]. In this paper, we assume that the offset (zero-current) correction is possible. The correction can be done by switching off the measured current. In case measured current cannot be switched off, zero output can be measured at the sufficient distance, in which the effect of the current is negligible. In case zero calibration is not possible at all, the long-term stability of the sensor offset will be given by offset stability of the flipped sensors. The offset stability of the flipped KMZ 51 is in the range of $2 \mathrm{nT} /{ }^{\circ} \mathrm{C}$ which corresponds to $0.1 \mathrm{~mA} /{ }^{\circ} \mathrm{C}$. It should be noted that the sensor offset can be seriously influenced by strong field gradients such as from permanent magnets in the vicinity of the sensor.

The results of zero output measurements are shown in Figures 5 and 6 by the blue (circle) plots, zero readings.

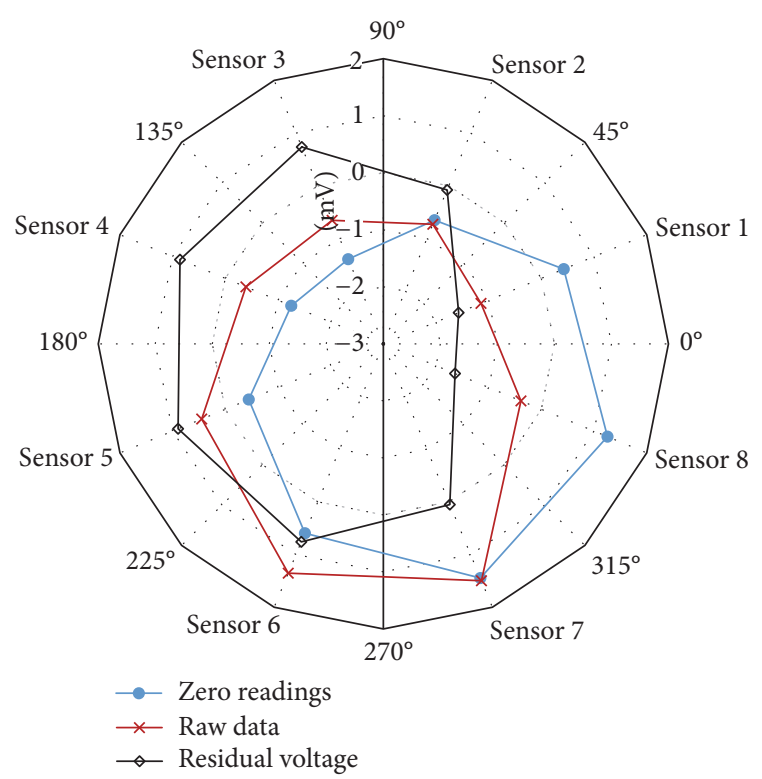

FIgURE 6: The residual output voltage of the AMR sensors with the off-center current at the angle $0^{\circ}$.

Next, the measured current was applied in different positions inside the circular hole. The most interesting positions are at the edge of the hole where the error is maximal. As an example, the results of two positions on the edge of the hole (angle $\alpha=235^{\circ}$ and $\alpha=0^{\circ}$, resp., defined in Figure 3) are shown in Figure 5 and Figure 6, respectively, by the red (cross) plots, raw data.

The residual voltages were calculated as a subtraction of raw data and zero readings of AMRs. Then they were caused only by the off-center measured current. The position of the current ( $\alpha$ and $r$ ) was estimated from these residual voltages using formula (3). The residual voltages are shown in Figures 5 and 6 by the black (diamond) plots.

The SW written in LabVIEW ${ }^{\mathrm{TM}} 2015$ environment was used to determine the position of the current. The included Nonlinear Curve Fit.vi uses formula (3) as a fitting model $f(x, a)[18]$ for all eight sensors. The VI (virtual instrument) uses the Levenberg-Marquardt algorithm to determine two common parameters-displacement and angle ( $\alpha$ and $r$ )-from eight formulas. The estimated current from the device (derived from compensation current) serves as an independent value $X$ and residual voltages are dependent values $Y$.

5.2. Calibration of the Device. The main part of improving the accuracy of the whole device is the calibration process. It consists of two steps, measuring the zero readings (caused by the external magnetic field) and measuring the current in several positions to map the error to the different position of the measured current.

Measurement of zero readings was described in the previous paragraph. Next, the well-known measured current is switched on and applied in several positions inside the hole. It is important to keep the device in a stable position during 


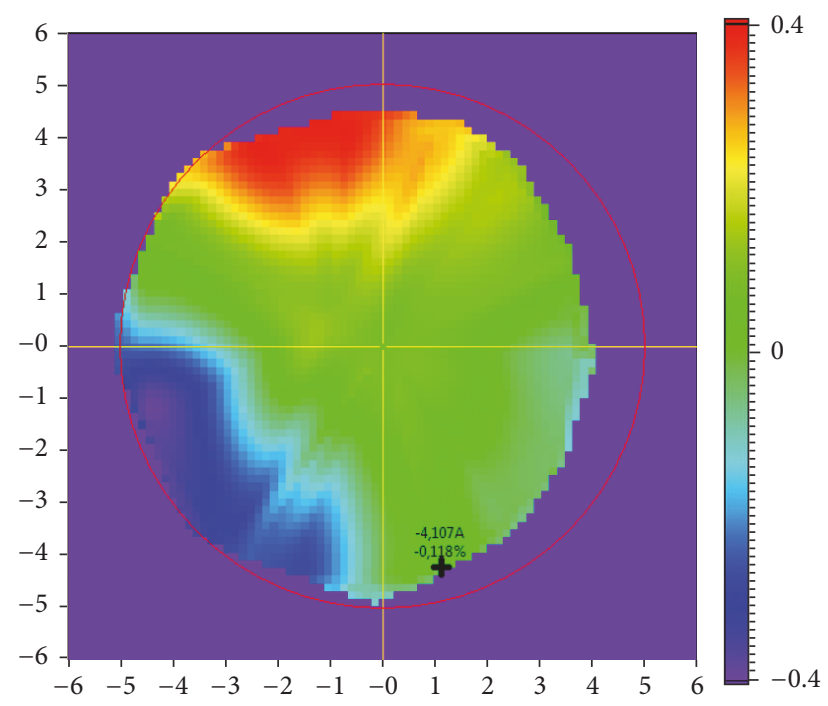

FIGURE 7: Lookup table in memory to correct the measurement error (red circle represents the hole, $x$ - and $y$-scales are in $\mathrm{mm}, z$-scale is in percentage); black cross represents actual position of current with the value and error.

the calibration. Calibration points should cover location close to the center of the hole and the positions where the offcenter error is the greatest, for example, around the edge of the hole. During this process, the position is determined from the residual voltages (zero readings subtracted from raw data) and stored in memory as well as the magnitude of the measurement error according to (7).

These points in memory represent a lookup table (position versus error) for next measurements where the value of the error is subtracted from the estimated current. More calibration positions mean better knowledge about the distribution of the off-center error but many points bring longer calibration process. The interpolation is used to better spatial resolution of the lookup table.

The error correction for the positions between the calibration points are calculated by Interpolate 2D Scattered.vi [19] with the cubic method in our SW in LabVIEW 2015 environment. The graphical representation of an example of the lookup table is shown in Figure 7. This lookup table was constructed from 9 calibration points: one in the center of the hole and eight on the edge of the hole close to particular AMRs. The other points are interpolated by the software.

5.3. Correction Process. First of all, zero correction is made if necessary. The first estimate of the measured current is calculated from the compensation current as if the current was in the middle of the sensor. The position of the current is then determined from the residual voltages and the estimated current as was described in Section 5.1. The displacement and angle are used to look up memory for the error corrections. The correction of the current estimate is made by subtracting the errors from the lookup table. The results are shown in Figure 4 by the blue (circle) plot. The measurement positions are the same as for uncorrected measurement, red plot in the same figure. The uncorrected error is in the range of approx. $\pm 0.4 \%$ and after correcting the error decreases to $\pm 0.08 \%$ (800 ppm).

\section{Conclusion}

The paper presents a new calibration method which allows improving the current estimation from the circular array of AMR sensors. The sources of the measurement errors are the nonidentical basic parameters of used sensors such as sensitivity and compensation coil factors in combination with the off-centered position of the measured current conductor. A large part of the off-center error can be suppressed by the newly developed calibration and correction process. The offcenter error was reduced more than 5 times in our device by using the correction lookup table. Our new method does not require magnetic shielding. The transducer can be recalibrated by the user.

The only limitation of the new method is the necessity of performing zero measurements. If the measured current cannot be switched off, zero measurements should be done at a sufficient distance from electric currents. For the zero measurements, the direction of the transducer with respect to the Earth's field should be kept same as for the current measurement. This limitation is a challenge for the future development of the device.

\section{Conflicts of Interest}

The authors declare that they have no conflicts of interest.

\section{Acknowledgments}

This work was supported by the Grant Agency of the Czech Republic through Project "New Methods for the Measurement of Electric Currents" (GACR 17-19877S).

\section{References}

[1] P. Ripka, "Electric current sensors: A review," Measurement Science and Technology, vol. 21, no. 11, Article ID 112001, 2010.

[2] P. Ripka, K. Draxler, and R. Styblíková, "DC-compensated current transformer," Sensors, vol. 16, no. 1, article no. 114, 2016.

[3] Y.-P. Tsai, K.-L. Chen, Y.-R. Chen, and N. Chen, "Multifunctional coreless hall-effect current transformer for the protection and measurement of power systems," IEEE Transactions on Instrumentation and Measurement, vol. 63, no. 3, pp. 557-565, 2014.

[4] J. Wang, D. Si, T. Tian, and R. Ren, "Design and Experimental Study of a Current Transformer with a Stacked PCB Based on B-Dot," Sensors, vol. 17, no. 4, p. 820, 2017.

[5] F. V. B. De Nazaré, M. M. Werneck, R. P. De Oliveira, D. M. Santos, R. C. Allil, and B. A. Ribeiro, "Development of an optical sensor head for current and temperature measurements in power systems," Journal of Sensors, vol. 2013, Article ID 393406, 2013.

[6] V. Frick, L. Hébrard, P. Poure, F. Anstotz, and F. Braun, "CMOS microsystem for AC current measurement with galvanic isolation," IEEE Sensors Journal, vol. 3, no. 6, pp. 752-760, 2003. 
[7] M. Blagojevic, U. Jovanovic, I. Jovanovic, D. Mancic, and R. S. Popovic, "Realization and optimization of bus bar current transducers based on Hall effect sensors," Measurement Science and Technology, vol. 27, no. 6, Article ID 065102, 2016.

[8] K.-L. Chen and N. Chen, "A new method for power current measurement using a coreless Hall effect current transformer," IEEE Transactions on Instrumentation and Measurement, vol. 60, no. 1, pp. 158-169, 2011.

[9] P. Mlejnek, M. Vopálenský, and P. Ripka, "AMR current measurement device," Sensors and Actuators A: Physical, vol. 141, no. 2, pp. 649-653, 2008.

[10] L. Di Rienzo and Z. Zhang, "Spatial harmonic expansion for use with magnetic sensor arrays," IEEE Transactions on Magnetics, vol. 46, no. 1, pp. 53-58, 2010.

[11] T. Y. Poon, N. C. F. Tse, and R. W. H. Lau, "Extending the GMR current measurement range with a counteracting magnetic field," Sensors, vol. 13, no. 6, pp. 8042-8059, 2013.

[12] C. Reig, M.-D. Cubells-Beltrán, and D. R. Muñoz, "Magnetic field sensors based on Giant Magnetoresistance (GMR) technology: Applications in electrical current sensing," Sensors, vol. 9, no. 10, pp. 7919-7942, 2009.

[13] M. Bichurin, R. Petrov, V. Leontiev, G. Semenov, and O. Sokolov, "Magnetoelectric Current Sensors," Sensors, vol. 17, p. 1271, 2017.

[14] D. Lee, M. Eissa, A. Gabrys et al., "Fabrication and Performance of Integrated Fluxgate for Current Sensing Applications," IEEE Transactions on Magnetics, no. 99, 2017.

[15] R. Weiss, R. Makuch, A. Itzke, and R. Weigel, "Crosstalk in circular arrays of magnetic sensors for current measurement," IEEE Transactions on Industrial Electronics, vol. 64, no. 6, pp. 4903-4909, 2017.

[16] P. Mlejnek and P. Ripka, "AMR Yokeless Current Sensor with Improved Accuracy," Procedia Engineering, vol. 168, pp. 900903, 2016.

[17] “Philips: KMZ51 Magnetic field sensor (datasheet)," 2000 http:// pdf.datasheetcatalog.com/datasheet/philips/KMZ51_3.pdf.

[18] "Nonlinear Curve Fit, Online LabVIEW Help," http://zone.ni.com/ reference/en-XX/help/371361H-01/gmath/nonlinear_curve_fit/.

[19] “Interpolate 2D Scattered, Online LabVIEW Help," http://zone .ni.com/reference/en-XX/help/371361H-01/gmath/interpolate 2d_scattered/. 


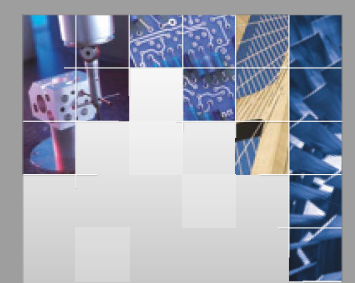

\section{Enfincering}
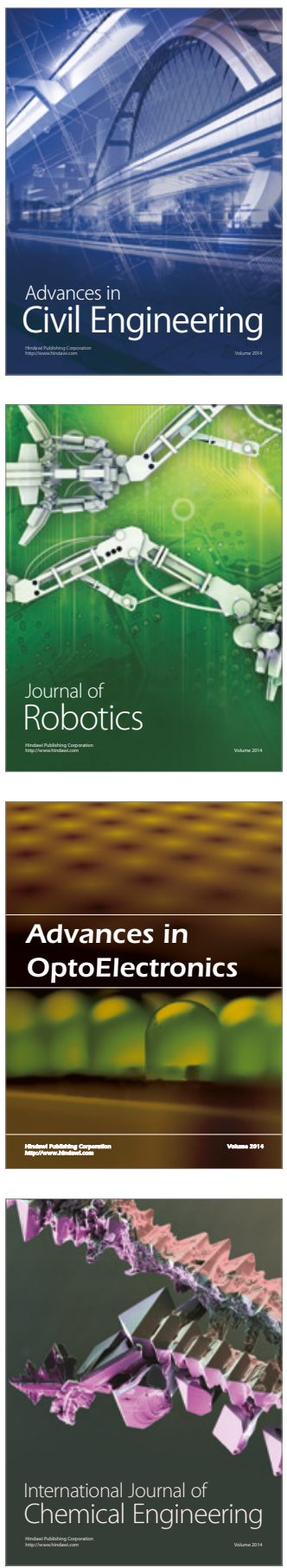

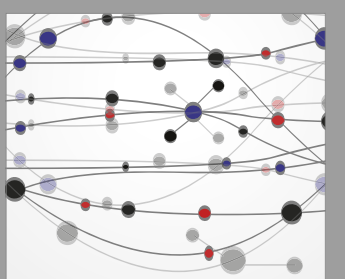

The Scientific World Journal

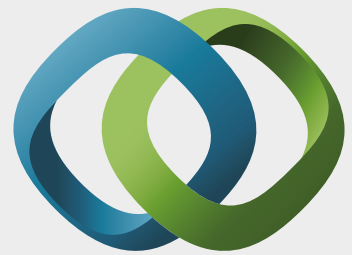

\section{Hindawi}

Submit your manuscripts at

https://www.hindawi.com
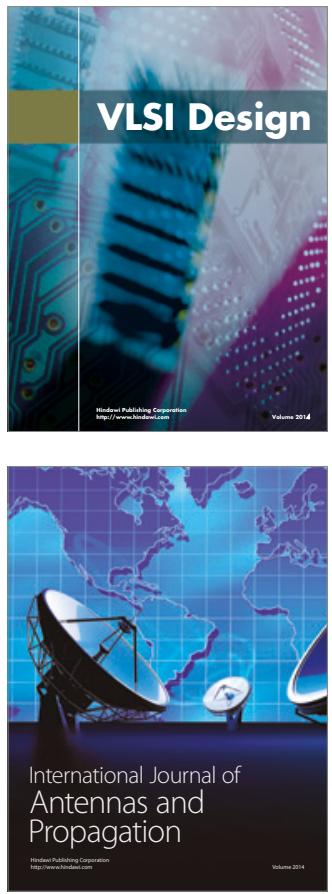

\section{Rotating}

Machinery
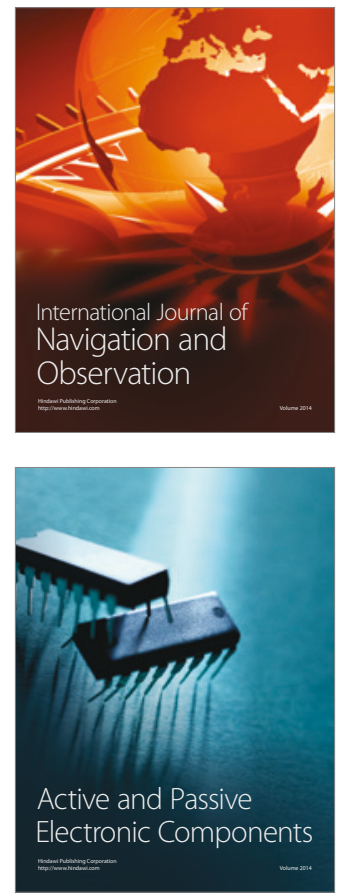
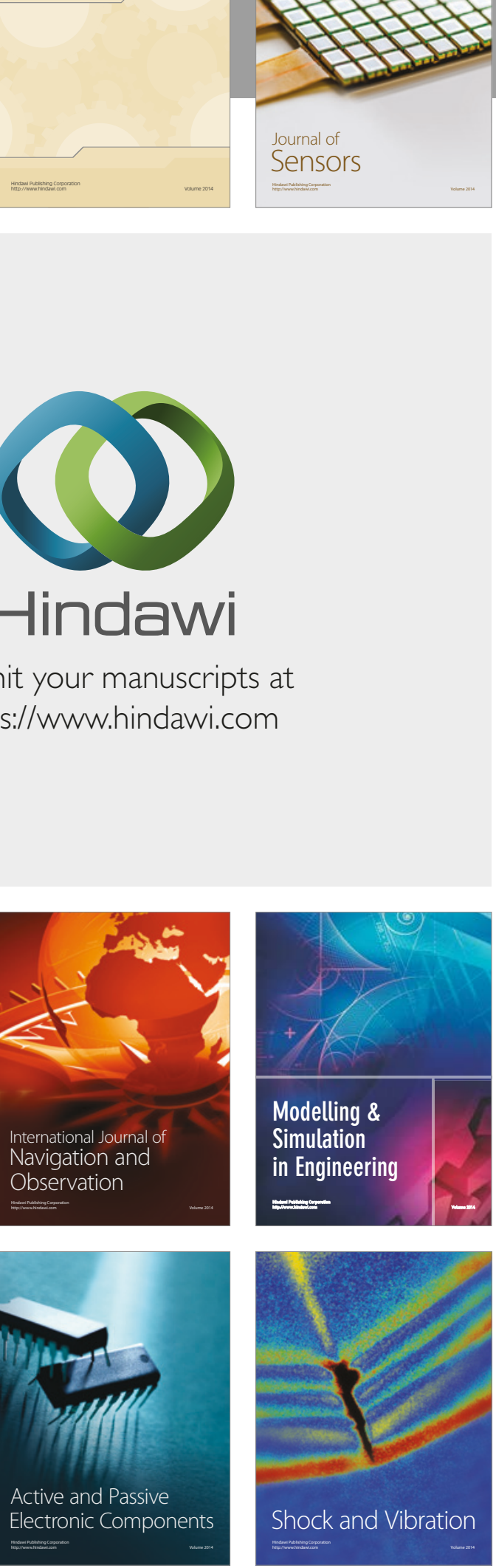
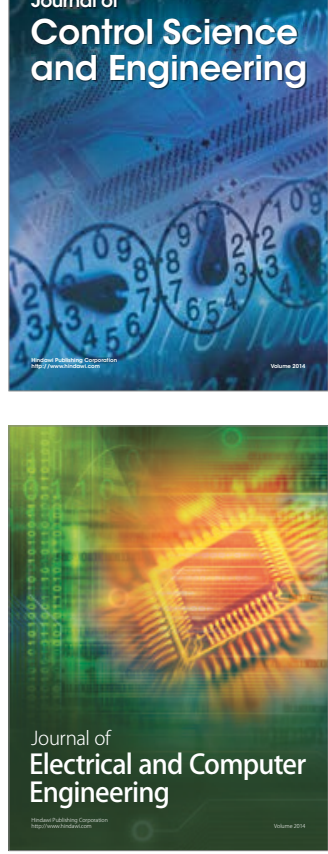

Distributed

Journal of

Control Science

and Engineering
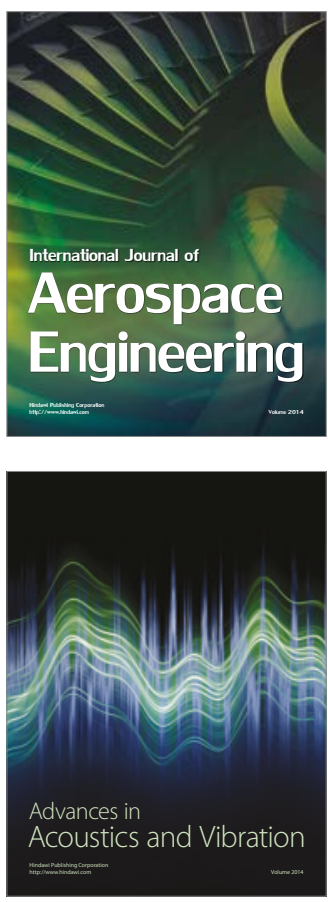

Sensor Networks 